\title{
Erasure Encoding: A Technique to enhance performance over Spray and Wait Protocol
}

\author{
Gireesh Kumar Sahariya \\ M.Tech(CSE) \\ LNCT Bhopal(M.P.) India
}

\author{
Vineet Richhariya, PhD \\ HOD (CSE) \\ LNCT Bhopal(M.P.) India
}

\author{
Vikram Rajput \\ Assistant Professor(CSE) \\ LNCT Bhopal(M.P.) India
}

\begin{abstract}
Delay Disruption Tolerant Networks are the network comes under the Wireless network protocol type which trend and used to provide a high security in network such as military area and other required government phases. The investigation research is already carried out to determine the best algorithm out of available algorithm which provides high efficiency in terms of its required parameter. In this paper we have discussed about the spray and wait algorithm which is the latest and reliable trend in terms of secure protocol in DTN and limitation observed as the packet size increases, it doesn't transform the packet properly. In order to enhance performance for large packet we have introduced Erasure encoding based spray and wait technique which improved the result in terms of required parameter.
\end{abstract}

\section{Keywords}

Delay tolerant networks (DTNs), Routing, store carry and forward.

\section{INTRODUCTION}

Due to the rigorous operation condition and the lack of continuous network connectivity, there is a large spectrum of application which prioritizes eventual message delivery over the message delay. Network serving these kind of application are generalized as Delay Tolerant Network (DTN).kavin fall, a member of intel research group introduced the Delay Tolerant network in which they provide a network architecture and an application interface to synchronize forwarding of messages within a partition based network in which topology changes continuously and provides long delays. It is a infrastructure less wireless network. It also experiences frequent and higher duration partitions due to nodes in DTN are intermittently connected. DTN network provides no guarantee that a path from source to destination will remain same at every time instance by which we can end that two nodes will never exist in a one connected portion of the network.

\section{DIFFERENTROUTING STRATEGY IN DELAY TOLERANT NETWORK}

Many Researchers has been extensively focused and discussed about routing in Delay tolerant network, which is the main part in DTN. The major purpose of routing is to provide the maximum possibility of deliver messages. Routing consists of a series of self-sufficient, local forwarding decision. This is based on the present connectivity and prediction of future connectivity information . Can also say node mobility needs to be broken in order to send a message to its target.

There mobility is take advantage of in order to expandability, while here it is used to beat the lack of end to end connectivity. It also depending on the numeral of copies of a lone message that may coexist in the system.Network
Topology in DTN can be roughly classified into two categories as Deterministic and stochastic routing. In deterministic case the future network 'state/topology and/or its characteristics are predictable. The main thought in computing the optimal route from source to a destination in deterministic routing protocol is based on complete information or predictable information about nodes future mobility patterns and links availability among them. Oracles based, Link state based, Space time based, and tree based are the example of deterministic routing approaches And Stochastic routing where no exact knowledge of future network topology is assumed. When there is no knowledge about nodes mobility patterns obtained via deterministic predictions or historic information stochastic routing mechanisms need to be used. Epidemic routing, history based, model based, coding based and flooding based are the example of stochastic routing approaches.

T.Spyropoulosetal.Introduces two types of routing that is single-copy scheme and multi-copy scheme. In single copy routing scheme there is only one guardian for each message all over the network. When the present guardian forwards the copy to asuitable next hop, this turn into the message's new guardian and so on. The present guardian forwards the message until attained its destination. He is also introduces other single copy scheme for example randomized routing algorithm, utility based routing and as seek and focus routing algorithm.

Alternatively, multiple copy (or multi-copy) routing schemes the node carrying the message sends a copy to each encountered node. This is frequent until the target receives the message. In this case, the contacts are assumed to be totally opportunistic. PROPHET protocol, Epidemic Routing protocol, and Spray\&Wait routing protocol are the use of this strategy. Due to low delivery ratio and long delay of the single-copy routing scheme, the multi-copy outing is the mainstream routing.

\subsection{DTNRouting Protocol Strategy can be Classified Into Three Broad Categories and They are Flooding, Replication and Forwarding.}

Many challenges affect the routing in DTN such as the changing network topology, Low delivery ratio and high delay. The problem can be mitigated by using different routing strategy. Many research interests focus on developing new approaches for routing in delay tolerant network environment. These routing schemes generally use the storecarry-and forward approach, where intermediate nodes keep message until encounter other nodes to set up new links in the path to the destination. 


\subsubsection{Flooding}

Flooding families are extended the number of copies of each message to a group of nodes, this node works like relays. The relays stock up the message pending they connect with the target, at which the message is deliver. With this strategy the flooding families increase the packet delivery ratio, also try to decrease the packet delivery delay. Epidemic routing, MaxProp, ProPhet are the example of Flooding routing Protocol. Awareness about the network helps in deciding the best next hop. It is possible that the network has no knowledge about the network.

In such Scenario, all nodes are made relay nodes. Such Schemes are called epidemic routing scheme. The epidemic routing is the simplest and earliest routing scheme for DTN. The basic concept of Epidemic routing is when two nodes encounter, they exchange messages each other and message will be propagated to the destination. It is based on Flooding delivery and the data delivery results in inefficient use of the network resources such as bandwidth, power and buffer space at each node. Fig: 1 in which the all nodes of network have the packet, where $A$ and $G$ are source and destination.

MaxProp Routing protocol uses the same routing as epidemic but improves buffer management. It is base on the agenda of packets transmit to other peers and the schedule of packets to be drop in delay tolerant networks.

PRoPHET is a probabilistic protocol for routing in irregularly connected networks that is more sophisticated, using history of node contacts and transitivity to improve performance .

\subsubsection{Replication}

Replication scheme insert multiple copies, or replicas of message into the network in order to increase the probability of message delivery that one of them will finds its way to the destination. This scheme further separated into two classes based on the no. of replicas created: Quota based and flooding based. In quota based protocol purposely limit the no. of replicas of message in the network. The quota of message is decided based upon certain quota allocation function. This function may be static or dynamic. Spray and wait, And EBR are the example of replication routing scheme. Quota allocation function i.e. binary value is used by spray and wait routing protocol in which it consist of two phase: spray phase and wait phase. In first phase it spread the sufficient no. of message copies. The nodes transport a message copy does direct show in the wait phase if the destination is not reach in the spray stage.

In encounter based routing protocol(EBR) is also a quotabased routing protocol. It minimizing network resource usage by limits the number of replicas of any massage in the network. EBR maintains an encounter value for each node, which is the average number of encounter with other nodes during an observation

\subsubsection{Forwarding}

In the Forwarding routing strategy, keep a single-copy message in the network and attempt to forward that copy through successive intermediate node to the destination. It takes more traditional approach on the basis of network topology knowledge to routing data in a DTN. And it select the best path to transmit message from node to node. A best route can be found by using Location-based routing, Per-hop routing, per-contact routing, and hierarchical routing protocol. Forwarding routing protocol such as MED, MEED [11] and Simbet
Jain has introduced many routing algorithms, based on knowledge oracles. They introduce the Minimum Expected Delay (MED) protocol [11] that is based on the prospect contact schedule. Also Jones get better work by introducing Minimum Estimated Expected Delay (MEED) [11], in which the supposed delay is

intended using the examiner contact history. Sliding window is used by every node in the network for tracing the connection and disconnection time of every contact.

Our proposed algorithm in order to have a process message on DTN we have combined our QBMR technique with erasure encoding Technique and performing our proposed solution on large data block processing mechanism in quota based multicast routing protocol.

\section{PROBLEM FORMULATION}

As per study the scenario of spray and wait algorithm provide an efficient routing where the packet delivery ration is maximum as it transmit the highest number of packet which increase its probability for packet delivery. Still upon this advantage its having some deficiency such as it doesn't not follow the large data packet size to deliver in the network. Large packet may overflow buffer size due to which the packet delivery ratio and delay for the large packet size is not suggested. The existing algorithm having the high efficiency but as such for delivering large data packet size with require algorithm to further process with the spray and wait routing protocol.

\section{PROPOSED SCHEME 4.1 Erasure coding based forwarding Algorithm}

Our erasure-coding based forwarding algorithm can be understood as an enhancement to the simple replication algorithm (srep). A simple replication strategy in which identical copies of the message are sent over the first $r$ contacts. Here, $r$ is the replication factor. Only the source of the message sends multiple copies. The relay nodes are allowed to send only to the destination; they cannot forward it to another relay. This leads to small overhead as the message flooding is controlled to take place only near the source. This class of forwarding algorithms is also known as the twohop relay algorithm [4, 5]. There is a natural trade-off between overhead ( $r$ ) and data delivery latency. A higher $\mathrm{r}$ leads to more storage/transmissions but has lower delays.

In srep with a replication factor $r$, the source sends $r$ identical copies over $r$ contacts and relays are only allowed to send directly to the destination. In the erasure-coding based algorithm, we first encode the message at the source and generate a large number of code blocks. The generated code blocks are then equally split among the first kr relays, for some constant $\mathrm{k}$. In comparison with step, this approach uses a factor of $\mathrm{k}$ more relays and each relay carries a factor of $1 / \mathrm{k}$ less data. However, the number of bytes generated are $\mathrm{rM}$, the same as the number of bytes generated by srep (r). Now by definition of erasure coding (with rate $r$, message size $M$ ), the message can be decoded at the destination if $1 / \mathrm{r}$ of the generated code blocks are received. Since code blocks are divided equally among $\mathrm{kr}$ relays, the message can be decoded as soon as any k relays deliver their data if we assume that no code blocks are lost during transmissions to and from a relay. When $\mathrm{k}=1$, the erasure coding approach has the same effect as the simple replication approach, which is, to use the first $r$ relays and to each carry a copy of the original message. 


\subsection{Benefits of erasure coding in forwarding}

In simple replication, $r$ relays are used to improve the delay performance. The erasure-coding based approach, instead, utilizes kr relays for the same amount of overhead. Therefore, one can expect that the chances of at least some relays having low delays are higher, compared to using only $r$ relays. At the same time, erasure coding requires at least $\mathrm{k}$ relays to succeed (instead of 1 in srep) before the data can be reconstructed. Therefore, if the number of such low-delay relays is larger than $\mathrm{k}$, the erasure-coding based approach will successfully deliver the message with a lower delay than simple replication. The main observation is that if $\mathrm{k}$ is large, the delay distribution converges to a constant.

Therefore, with the erasure-coding based approach, one can be almost assured of a constant delay.

Erasure coding based forwarding Algorithm

\section{Algorithm:}

Input: message block of size $\mathrm{m}=50 \mathrm{mb}$.

Output: Packet transfer and received at receiver end.

Step 1

Begin:

Step 2

If $\mathrm{M}=50 \mathrm{mb}$;

Step 3

Checking the data packet size and verify for the transmission.

Step 4 if $M>5 m b$

\{

erasure encoding scheme on Message size using accepted packet size.

$\mathrm{N}=(\mathrm{M} \times \mathrm{R}) / \mathrm{b}$ equal size code blocks

\}

Step 5

We are considering replication factor $\mathrm{r}=3$, Apply spray and wait algorithm to transfer the packet.

Step 6

If node $\mathrm{j}$ is a multicast receiver of message $\mathrm{m} \& \&$ if $(\mathrm{m}($ size $)==\mathrm{N} / \mathrm{R})$

Step 7

At destination at least $\mathrm{N} / \mathrm{R}$ code block are necessary to reconstruct the message

Step 8

End

\section{RESULT ANALYSIS}

In order to work with the algorithm and simulate them in experimental setup, we have used one simulator for the proposed and existing system simulation. Where we have performed work with the spray and wait as a existing system protocol and the erasure based spray and wait as a proposed system protocol, such that the system perform a output based on the number of parameters :
1. Relay in packets : it is the unit using which can calculate the packet relay while communication occur in particular time duration.

2. Overhead: this is the unit to calculate the traffic and communication overhead occur using the algorithm at the time of simulation.

3. Probability: this is the condition at the packet delivery using which we measure a stability of an algorithm, that it transfer the packets.

4. Latency: A unit to verify or monitor the time and measure algorithm efficiency.

Upon monitoring the performance using different two algorithms we have come up with the following result set and observe the following result.

Table 1: Result comparison using relay and latency average

\begin{tabular}{|c|c|c|}
\hline & $\begin{array}{l}\text { Spray \& } \\
\text { Wait }\end{array}$ & Erasure-Spray \& Wait \\
\hline Relay & 677 & 438 \\
\hline $\begin{array}{l}\text { Latency } \\
\text { Avg }\end{array}$ & 1557 & 1419 \\
\hline
\end{tabular}

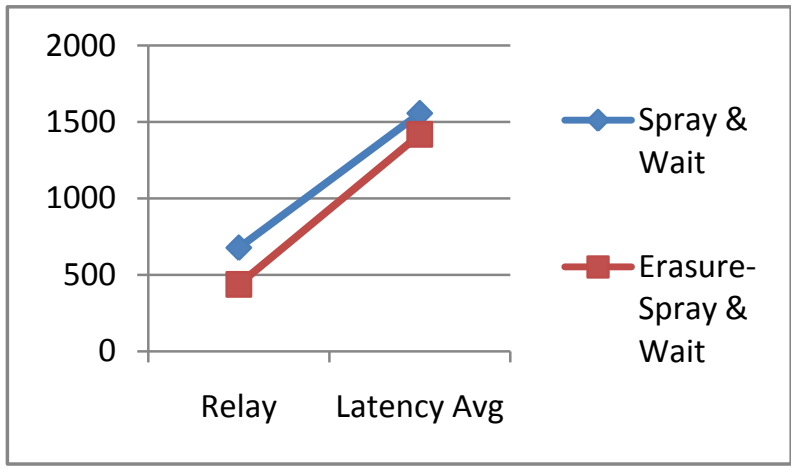

Figure 1: Packet relay and latency comparison graph

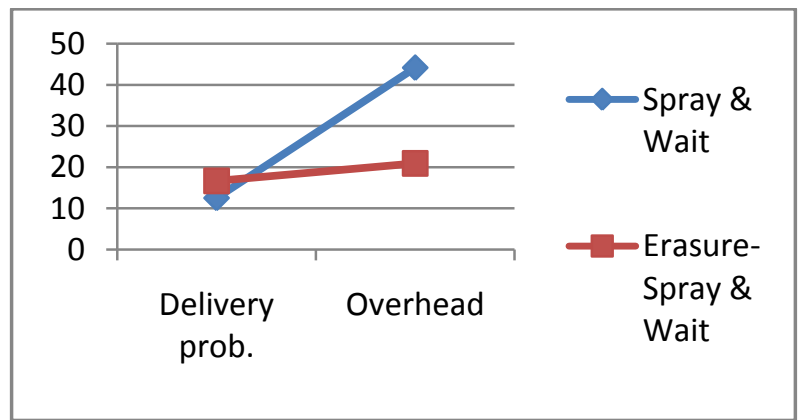

Figure 2: Delivery probability and overhead ration comparison.

Table 2: Different result observed Probability and overhead.

\begin{tabular}{|l|r|r|}
\hline & Spray \& Wait & Erasure-Spray \& Wait \\
\hline Delivery prob. & 12.5 & 16.67 \\
\hline Overhead & 44.13 & 20.9 \\
\hline
\end{tabular}




\section{CONCLUSION}

DTN communication technique is always a required technique where a secure communication is always required such as security area, secure network. Thus a technique required where the secure and high efficient algorithm required to be work. In our paper we have implemented Spray and wait algorithm which is the existing technique for communication and the further enhancement erasure encoding being embedded to improve the performance. According to the result observed, we identified that the technique perform a good performance in terms probability ration, overhead, Latency average and Relay in the packet network.

Our further work will be on working with Quality of services encryption module in the network using our algorithm.

\section{REFERENCES}

[1] A. McMahon and S. Farell, "Distruption-Tolerant Networking," IEEE Internet Computing, vol.13, no.6, pp. 82-87, Nov.2009.Khabbaz, M.,Assi, C., \& Fawaz, W. (2011).

[2] A. Vahdat and D. Becker, "Epidemic Routing for Partially Connected Ad Hoc Networks," Duke university, Technically Report CS-200006,Apr. 2000.'

[3] A. Lindgren, A. Doria, and O. Scheln. "Probabilistic Routing in Intermittently Connected Networks." LNCS, Springer, vol. 3126, pp.239-254, 2004.

[4] Delay-Tolerant Networking Research group (DTNRG), http://www.dtnrg.org

[5] IPN Special Interest Group (IPNSIG), http://www.ipnsig.org/

[6] K. Fall, "A Delay-Tolerant Network Architecture for Challenged Internets," in Proc. ACM SIGCOMM Conf., pp. 27-34, Aug. 2003.

[7] Khabbaz, M., Assi, C., \& Fawaz, "Distruption-Tolerant Networking: A Comprehensive Survey on Recent Development and Persisting Challenges. Communication Surveys \& Tutorials", IEEE, pp(99), 1-34.

[8] J. Burgess, B. Gallagher, D. Jensen, and B. N. Levine, "MaxProp:Routing for Vehicle-Based Distruption-
Tolerant Networks," in Proc.IEEE INFOCOM Conf., pp.1-11, Apr. 2006.

[9] McMahon and S. Farrell, "Delay- and DisruptionTolerant Networking," IEEE Internet Computing, vol. 13, no.6, pp. 82-87, Nov.2009.

[10] S. C. Lo, M. H. Chiang, J. H. Liou, and J.S. Gao, "Routing And Buffering Strategies in Delay Tolerant Networks: Survey and Evaluation," on Proc. IEEE ICPP Workshop, Sept. 2011

[11] E. P. C. Jones, L. Li, And P. A. S. Ward, "Practical Routing in Delay tolerant Networks," IEEE Trans. Mobile Computing, vol. 6, no.8 pp.943-959, Aug. 2007.

[12] R. J. D'Souza and J. Jose,'Routing Approaches in Delay Tolerant Networks: A Survey," Intl. Journal of Computer Application, vol. 1, no.17, pp. 8-14, 2010."

[13] Z Zhang"Routing In Intermittently Connected Mobile Ad Hoc Networks and Delay Tolerant: Overviews and Challenges," in Proc. IEEE communication survey\&tutorial, $1^{\text {st }}$ quarter 2006.

[14] T. Abdelkader, K.Naik, A. Nayak, N.Goel, and V. Srivastava," SGBR: A Routing Protocol for Delay Tolerant Networks Using Social Grouping,"in ieee,2012.

[15] T. Spyropoulos, K. Psounis, and C.S. Raghav,"SingleCopy Routing in Intermittently Connected Mobile Networks,"in IEEE 2004

[16] Samuel C. Nelson, Mehedi Bakht, Robin Kravetsa Albert F Harris "Poster Abstract: Encounter-Based Routing in DTNs".

[17] Thrasyvoulos Spyropoulos, Konstantinos Psounis and Cauligi S. Raghavendra, " Spray and Focus: Efficient Mobility-Assisted Routing for Heterogeneous and Correlated Mobility", IEEE 2007.

[18] Elizabeth Daly and Mads Haahr, Social Network Analysis Routing in Disconnected Delay-Tolerant MANETs 